\title{
Adsorption Behavior of Germanium(IV) on Branched-saccharide Chitosan Derivatives
}

\author{
Yoshinari INUKAI, Yasuhiko KAIDA and Seiji YASUDA
}

Kyushu National Industrial Research Institute, AIST, MITI, 807-1, Shuku-machi, Tosu, Saga 841, Japan

\begin{abstract}
Branched-saccharide-chitosan resins and beads selectively adsorbed germanium(IV) from aqueous solutions containing semimetals over the range of weakly acidic to basic media by a batch method. The chitosan derivatives had large adsorption capacities of up to about $2 \mathrm{mmol} \mathrm{g}^{-1}$. On the other hand, by a column method, the chitosan derivatives adsorbed both germanium(IV) and boron. However, when a mixture of germanium(IV) and boron was applied to the column, boron, initially adsorbed on the column, was gradually substituted by germanium(IV), and was finally eluted. The germanium(IV), adsorbed on the column, was almost quantitatively recovered by elution with hydrochloric acid.
\end{abstract}

Keywords Germanium(IV), chitosan, saccharide, semimetal

Germanium compounds have been widely used in various fields of industry, for example as a catalyst in the production of polyesters. The separation and recovery of germanium(IV) are important because of its high cost. However, germanium(IV) cannot be adsorbed by conventional chelating resins for metal cations, because it dissolves as oxoacid, $\mathrm{Ge}(\mathrm{OH})_{4}$, and oxoaions, $\mathrm{GeO}(\mathrm{OH})_{3}{ }^{-}$and $\mathrm{GeO}_{2}(\mathrm{OH})_{2}{ }^{2-}$, in aqueous solutions. $^{1} \quad$ A strongly basic anion-exchange resin can adsorb germanium(IV $)^{2}$, however it has no selectivity for germanium(IV) over anions ${ }^{3-5}$ On the other hand, it is well known that germanium(IV) forms complexes with polyol compounds ${ }^{6}$ or saccharides. ${ }^{6,7}$ Therefore, commercially available Sephadex gel$^{1}$ and $\mathrm{N}$-methylglucamine resins ${ }^{7-12}$ containing hydroxy groups are used for the preconcentration and separation of germanium(IV). However, the resins do not show high selectivity for germanium(IV) over boron, because they also strongly adsorb boron ${ }^{8,11,13-16}$

In previous studies ${ }^{17,18}$ we synthesized 1,2-dioland 1,3-diol-types polystyrene chelating resins to obtain novel adsorbents with high selectivity for semimetals. The 1,3-diol-type resins adsorbed only boron from semimetals solutions, such as germanium(IV) and tellurium(VI) solutions, in both batch and column methods. ${ }^{17}$ The 1,2-diol-type resins selectively adsorbed germanium(IV) over boron, tellurium(VI), arsenic(III) and silicon(IV) in a batch method. ${ }^{18}$ However, the adsorption capacities of the diol-types resins for boron and germanium(IV) were still smaller than, or comparable to, that of the commercially available $\mathrm{N}$-methylglucamine resin. ${ }^{10,14}$ On the other hand, chitosan and its derivatives have been widely applied as adsorbents for metal ions ${ }^{19}$, and some chitosan derivatives exhibit higher adsorption capacities for metal ions than conventional chelating resins. ${ }^{20,21}$ However, there have been no studies on the adsorption of semimetals, such as germanium(IV) and boron, on chitosan derivatives, except for a report about a polyaminated chitosan resin. ${ }^{22}$

In a preliminary communication ${ }^{23}$ we described the branched-saccharide-chitosan resins and beads, prepared from chitosan and saccharides. Germanium(IV) was selectively adsorbed on these resins and beads from aqueous solutions containing semimetals over the range of weakly acidic to basic media by a batch method. The chitosan resins had large adsorption capacities of up to about $2 \mathrm{mmol} \mathrm{g}^{-1}$. The adsorption mechanism is ascribed to complex formation between germanium( IV ) and hydroxy groups of the branched saccharide.

In the present study we added discussions on the adsorption rate and isotherms for germanium(IV) and tellurium( VI ) on the branched-saccharide-chitosan resins and beads by a batch method, and investigated the adsorption and desorption behavior of germanium( (V), tellurium(VI) and boron on the branched-saccharide-chitosan resins and beads by a column method.

\section{Experimental}

\section{Chemicals}

Chitosan (trade name: Kimitsu Chitosan LLWP) was donated by Kimitsu Chemical Industries Co., Ltd., Japan. The deacetylated degrees of Kimitsu Chitosan LLWP were determined to be $75-85 \%$ by colloidal titration. Chitosan beads (trade name: Chitopearl "Basic" AL-03, particle size $0.3 \mathrm{~mm}$ in 
diameter) were produced for the crosslinking of chitosan particles with ethylene glycol diglycidyl ether by Fuji Spinning Co., Ltd., Japan. ${ }^{22}$ Other reagents were reagent or higher grade, and were used without further purifications. All of the aqueous solutions were prepared with distilled and deionized water.

\section{Preparation}

Various branched-saccharide-chitosan resins and beads were prepared from chitosan, or chitosan beads, and saccharides, as shown in the preliminary communication. ${ }^{23}$

\section{Batch method}

About $50 \mathrm{mg}$ of the dried branched-saccharidechitosan resin or beads and $25 \mathrm{~cm}^{3}$ of the semimetal sample solution were shaken for $24 \mathrm{~h}$ in a screwed vial maintained at $25^{\circ} \mathrm{C}$. About $1 \mathrm{~cm}^{3}$ of the wet branched-saccharide chitosan beads was also used, when the adsorption rate of semimetal was measured. The initial concentration of semimetal in the sample solution was about $10 \mathrm{mmol} \mathrm{dm}$, except for in experiments of adsorption isotherms; $0.1 \mathrm{~mol} \mathrm{dm}^{-3}$ potassium chloride solution was used to maintain a constant ionic strength of the sample solution, except for in experiments of the adsorption rate. The initial $\mathrm{pH}$ was adjusted with addition of $0.1-0.5 \mathrm{~cm}^{3}$ diluted hydrochloric acid or aqueous sodium hydroxide. When the adsorption rate of semimetal was measured, ammonium chloride - ammonia buffer was used as the sample solution to maintain a constant $\mathrm{pH}$ value. The amounts of adsorbed semimetals were calculated from the differences between the concentrations of semimetals in the aqueous solutions before and after the adsorption, and expressed as mmol per $g$ of the chitosan resins or beads. The concentrations of semimetals were determined using an inductively coupled plasma (ICP) atomic-emission spectrometer (Seiko Instruments Inc., Japan; Model SPS 1200AR). The $\mathrm{pH}$ values at equilibrium after $24 \mathrm{~h}$ were measured with a pH meter (Horiba, Ltd., Japan; Model F-22).

\section{Column method}

The sample solution containing germanium(IV), tellurium(VI) and boron together was loaded on a column ( $5 \mathrm{~mm}$ in diameter), packed with about $1.3 \mathrm{~cm}^{3}$ of the wet branched-saccharide-chitosan resin or beads, at a flow rate of space velocity (SV) about $10 \mathrm{~h}^{-1}$ at room temperature. The concentration of each semimetal in the sample solution was about $1 \mathrm{mmol}$ $\mathrm{dm}^{-3}$. An ammonium chloride - ammonia buffer was used as the sample solution to maintain a constant $\mathrm{pH}$ value. The elution of the column was carried out by $1 \mathrm{~mol} \mathrm{dm}^{-3}$ hydrochloric acid at a flow rate of SV about $10 \mathrm{~h}^{-1}$.

In recycle experiments, 77 bed volumes of $1 \mathrm{mmol}$ $\mathrm{dm}^{-3}$ germanium(IV) solution, adjusted with a buffer to pH 8.0, were loaded on the branched-mannose

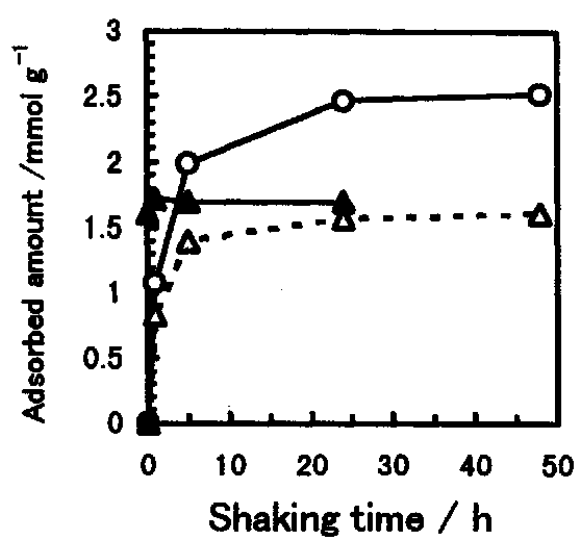

Fig. 1 Effect of the shaking time on the adsorption of germanium(IV) on the branched-mannose-chitosan resin (dried:O) and beads (dried: $\Delta$ )(wet:A) at $\mathrm{pH}$ 9.0-9.1.

chitosan resin column at a flow rate of SV about $10 \mathrm{~h}^{-1}$ at room temperature in each cycle. After being washed with deionized water, germanium(IV) in the column was eluted with 23 bed volumes of $1 \mathrm{~mol} \mathrm{dm}^{-3}$ hydrochloric acid at a flow rate of SV about $10 \mathrm{~h}^{-1}$. The column was reused after being washed with $1 \mathrm{~mol}$ $\mathrm{dm}^{-3}$ sodium hydroxide solution and deionized water in recycle experiments. The recovery of germanium(IV) was calculated from the ratio of the amount eluted with the eluent to the amount adsorbed on the column.

\section{Results and Discussion}

Adsorption rate and isotherms for germanium(IV) and tellurium( $V)$ on the branched-saccharidechitosan resins and beads

Branched-mannose-chitosan resin had the largest adsorption capacity for germanium(IV) from aqueous solution containing tellurium(VI) in the branchedsaccharide-chitosan resins and beads, as shown in the preliminary communication ${ }^{23}$. In addition, the adsorption rate and isotherms for germanium(IV) and tellurium(VI) on the branched-mannose-chitosan resin and beads by a batch method are discussed as follows.

Figure 1 shows the effect of the shaking time on the adsorption of germanium(IV) on the branchedmannose-chitosan resin and beads at $\mathrm{pH}$ 9.0-9.1. The adsorption of germanium(IV) on the wet beads reached equilibrium after $1 \mathrm{~h}$, and the adsorption capacity at equilibrium was about $0.078 \mathrm{mmol} \mathrm{cm}^{-3}$ (= $1.7 \mathrm{mmol} \mathrm{g}^{-1}$ ). For the dried chitosan resin and beads, however, $24 \mathrm{~h}$ was required to attain the adsorption equilibrium, and the adsorption capacities at equilibrium were about 2.5 and $1.5 \mathrm{mmol} \mathrm{g}^{-1}$, respectively. Such a decrease in the adsorption rate of germanium(IV) on the dried chitosan resin and beads is attributable to the slower intraparticle 


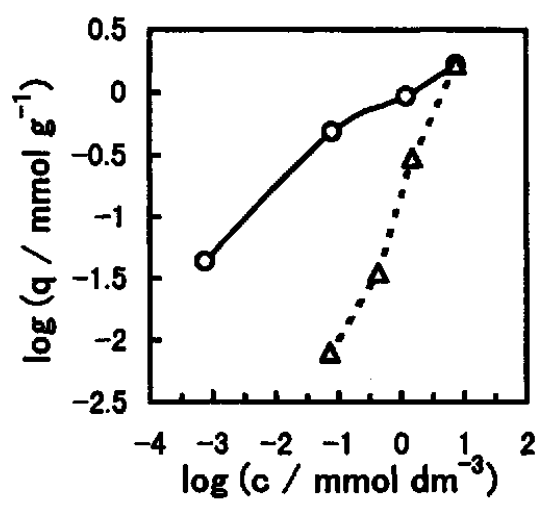

Fig. 2 Adsorption isotherms for germanium(IV)(O) and tellurium $(\mathrm{VI})(\Delta)$ on the branched-mannose chitosan resin at an equilibrium $\mathrm{pH}$ of $5.1-7.0$ at $25^{\circ} \mathrm{C}$.

q: amount adsorbed on the resin.

c: equilibrium concentration in the solution after $24 \mathrm{~h}$.

diffusion rate of germanium(IV) in the dried chitosan resin and beads than that in the wet chitosan resin and beads. Similar trends were observed in the adsorption of tellurium(VI), on the branched-mannose chitosan resin at $\mathrm{pH} 8.3$, and germanium(IV), on the branched-galacturonic acid-chitosan resin and beads at pH 8.9-9.1.

Figure 2 shows the adsorption isotherms for germanium(IV) and tellurium(VI) on the branchedmannose chitosan resin at an initial and equilibrium $\mathrm{pH}$ of 7.9-8.3 and 5.1-7.0, respectively, at $25^{\circ} \mathrm{C}$. The isotherms indicate that germanium(IV) was adsorbed on the resin more than tellurium(VI) in the low semimetal concentration range. Therefore, the separation of germanium(IV) from tellurium(VI) is presumed to be easy in the low semimetal concentration range. Similar trends were observed in the adsorption isotherms for germanium(IV) and tellurium(VI) on the branched-galacturonic acid and glucuronic acid chitosan resins at an initial and equilibrium $\mathrm{pH}$ of 7.8-8.3 and 5.6-7.7, respectively, at $25^{\circ} \mathrm{C}$.

Adsorption and desorption behavior of semimetals on and from the branched-saccharide-chitosan resins and beads columns

Figure 3 shows breakthrough curves for germanium( IV ), tellurium( VI) and boron in the branched-mannose chitosan resin column at $\mathrm{pH}$ 8.5. At first both germanium(IV) and boron were adsorbed on the column. The breakthrough points of germanium(IV) and boron were 140 and 80 bed volumes, respectively. The concentrations of boron in the effluent of $130-300$ bed volumes were higher than the initial concentration. This fact means boron, initially adsorbed on the column, was gradually substituted by germanium(IV), and was finally eluted.

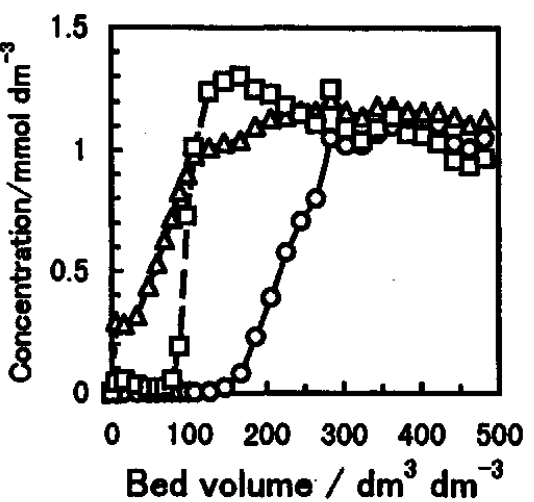

Fig. 3 Breakthrough curves for germanium $(\mathrm{I})(\mathrm{O})$, tellurium $(\mathrm{VI})(\Delta)$ and boron( $\square)$ in the branchedmannose chitosan resin column at pH 8.5.

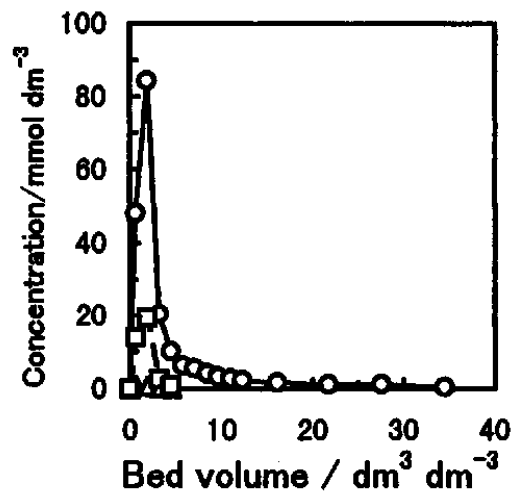

Fig. 4 Elution curves for germanium $(\mathrm{IV})(\mathrm{O})$, tellurium $(\mathrm{VI})(\Delta)$ and boron $(\square)$ with $1 \mathrm{~mol} \mathrm{dm}^{-3}$ hydrochloric acid in the branched-mannose chitosan resin column.

This substitution is due to the high separation factor of germanium(IV) to boron for the competitive adsorption of the branched-mannose chitosan resin by a batch method. $^{23}$ On the other hand, tellurium(VI) leaked immediately in spite of the low separation factor of germanium(IV) to tellurium(VI) for the competitive adsorption of the branched-mannose chitosan resin by a batch method. ${ }^{23}$ When only tellurium(VI) was loaded on the column, it did not leak immediately. Therefore, the adsorption of germanium(IV) and boron on the column seems to cause the leakage of tellurium(VI).

Figure 4 shows the elution curves for germanium(IV), tellurium(VI) and boron with $1 \mathrm{~mol}$ $\mathrm{dm}^{-3}$ hydrochloric acid in the branched-mannose chitosan resin column. Since no tellurium(VI) was adsorbed on the column, it was not eluted. A small amount of boron was eluted, because most of boron was substituted by germanium(IV). After all the elution of the column with hydrochloric acid mainly gave germanium(IV). Most of germanium(IV) was 
Table 1 Elution of germanium(IV), adsorbed on the branched-mannose chitosan resin column, with $1 \mathrm{~mol}$ $\mathbf{d m}^{-3}$ hydrochloric acid

\begin{tabular}{lccc}
$\begin{array}{l}\text { Cycle } \\
\text { No. }\end{array}$ & $\begin{array}{c}\text { Adsorbed }^{\mathbf{2}} \\
\text { Ge(IV)/ mg }\end{array}$ & $\begin{array}{c}\text { Eluted }^{\mathrm{b}} \\
\text { Ge(IV)/ mg }\end{array}$ & Recovery/\% \\
\hline 1 & 7.24 & 6.92 & 95.7 \\
2 & 7.24 & 6.58 & 90.9 \\
3 & 7.86 & 7.27 & 92.5
\end{tabular}

a. Sample volume was 77 bed volumes of $1 \mathrm{mmol} \mathrm{\textrm {dm } ^ { - 3 }}$ germanium(IV) solution at $\mathrm{pH} 8.0$

b. Eluent volume was 23 bed volumes of $1 \mathrm{~mol} \mathrm{dm}^{-3}$ hydrochloric acid.

eluted in the initial 10 bed volumes of the effluent.

Similar trends were observed in the adsorption and desorption of germanium(IV), tellurium(VI) and boron on the branched-glucuronic acid chitosan resin, the branched-xylose-chitosan resin and beads columns. The breakthrough points of germanium(IV), in the columns with almost same bed volume, decreased in the order of the branched-mannose chitosan resin $>$ the branched-glucuronic acid chitosan resin $\geqq$ the branched-xylose chitosan resin $>$ the branchedxylose chitosan beads. This order is the same as that of the adsorption capacities for germanium(IV) by a batch method. ${ }^{23}$ For the branched-xylose-chitosan resin and beads columns, most of germanium(IV) was eluted in less than 10 bed volumes of the effluent. However, for the branched-glucuronic acid chitosan resin column, 20 bed volumes of the effluent were required to elute most of germanium( $\mathrm{N}$ ) with hydrochloric acid.

The cycle of adsorption, washing, elution and washing steps was repeated 3 times in order to check the reproducibility of the branched-mannose chitosan resin column system in the adsorption and desorption of germanium(IV). The results are shown in Table 1. The germanium(IV), adsorbed on the column, was almost quantitatively eluted with hydrochloric acid in each cycle. That is, selective separation and a considerable concentration effect of germanium(IV) were achieved by utilizing the branched-mannose chitosan resin column.

We thank Messrs. Takeshi Adachi and Toyotaka Chinen for their experimental help, and Professor Toshio Matsuda (Tohwa University) for his helpful discussions.

\section{References}

1. A. Harada, T. Tarutani and K. Yoshimura, Anal. Chim. Acta, 209, 333 (1988).

2. D. A. Everest and J. E. Salmon, J. Chem. Soc., 1954, 2438.
3. D. A. Everest and W. J. Popiel, J. Chem. Soc., 1956, 3183.

4. D. A. Everest and W. J. Popiel, J. Chem. Soc., 1957, 2433.

5. D. A. Everest and W. J. Popiel, J. Inorg. Nucl. Chem., 6, 153 (1958).

6. P. J. Antikainen, Acta Chem. Scand., 13, 312 (1959).

7. P. J. Antikainen and E. Huttunen, Suomen Kemistilehti B, 46, 184 (1973).

8. S. Yasuda and K. Kawazu, Bunseki Kagaku, 37, T67 (1988).

9. S. Yasuda and K. Kawazu, Kyushu Kohgyo Gijyutsu Shikensyo Hohkoku, 1989, 2739.

10. S. Yasuda and K. Kawazu, Sep. Sci. Technol., 26, 1273 (1991).

11. U. Schilde and E. Uhlemann, React. Polym., 20, 181 (1993).

12. U. Schilde, H. Kraudeit and E. Uhlemann, React. Polym., 22, 101 (1994).

13. K. Yoshimura, R. Kariya and T. Tarutani, Anal. Chim. Acta, 109, 115 (1979).

14. S. Yasuda and H. Yamauchi, Nippon Kagaku Kaishi, 1987, 752.

15. U. Schilde and E. Uhlemann, Int. J. Mineral Process., 32, 295 (1991).

16. U. Schilde and E. Uhlemann, React. Polym., 18, 155 (1992).

17. S. Yasuda, Y. Inukai and $H$. Ohba, Bunseki Kagaku, 42, 713 (1993).

18. S. Yasuda, $Y$. Inukai and $H$. Ohba, Nippon Kagaku Kaishi, 1994, 221.

19. Japanese Society for Chitin and Chitosan (ed.), "Handbook of Chitin and Chitosan (in Japanese)", Gihohdoh Shuppan, Tokyo, 1995.

20. K. Inoue, K. Ohto, K. Yoshizuka, R. Shinbaru, Y. Baba and K. Kina, Bunseki Kagaku, 42, 725 (1993).

21. Y. Baba, Y. Kawano and H. Hirakawa, Bull. Chem. Soc. Jpn., 69, 1255 (1996).

22. Y. Kawamura, M. Mitsuhashi, $H$. Tanibe and $H$. Yoshida, Ind. Eng. Chem. Res., 32, 386 (1993).

23. Y. Inukai, Y. Kaida and S. Yasuda, Anal. Chim. Acta, 343, 275 (1997). 